\title{
Incidental Finding of a PSMA-Positive Pancreatic Cancer in a Patient Suffering from a Metastasized PSMA-Positive Prostate Cancer
}

\author{
Simon Sirtl ${ }^{1, *}$, Andrei Todica ${ }^{2}$, Harun Ilhan ${ }^{2}$, Michal Zorniak ${ }^{1}$, Peter Bartenstein ${ }^{2}$ and Julia Mayerle ${ }^{1}$ \\ 1 Department of Medicine II, University Hospital, 81377 LMU Munich, Germany; \\ michal.zorniak@med.uni-uenchen.de (M.Z.); julia.mayerle@med.uni-muenchen.de (J.M.) \\ 2 Department of Nuclear Medicine, University Hospital, 81377 LMU Munich, Germany; \\ andrei.todica@med.ui-muenchen.de (A.T.); harun.ilhan@med.uni-muenche.de (H.I.); \\ peter.bartenstein@med.uni-muenchen.de (P.B.) \\ * Correspondence: simon.sirtl@med.uni-muenchen.de; Tel.: +49-89-4400-72391
}

Citation: Sirtl, S.; Todica, A.; Ilhan, H.; Zorniak, M.; Bartenstein, P.; Mayerle, J. Incidental Finding of a PSMA-Positive Pancreatic Cancer in a Patient Suffering from a Metastasized PSMA-Positive Prostate Cancer. Diagnostics 2021, 11, 129. https:// doi.org/10.3390/diagnostics11010129

Received: 22 December 2020 Accepted: 14 January 2021 Published: 15 January 2021

Publisher's Note: MDPI stays neutral with regard to jurisdictional clai$\mathrm{ms}$ in published maps and institutional affiliations.

Copyright: $\odot 2021$ by the authors. Licensee MDPI, Basel, Switzerland. This article is an open access article distributed under the terms and conditions of the Creative Commons Attribution (CC BY) license (https:// creativecommons.org/licenses/by/ $4.0 /)$.

\begin{abstract}
An 82-year-old man suffering from prostate cancer that was scheduled for a radioreceptorligand therapy (RLT) presented with jaundice to our service. An abdominal ultrasound (US) revealed obstructive extrahepatic cholestasis due to a solid lesion located in the uncinate process of the pancreas. The Prostate Specific Membrane Antigen (PSMA) PET/CT prior to RLT showed multilocular PSMA positive tumor lesions in the lymph nodes, the lung and the pancreas. On request of the cancer board, an Endoscopic Ultrasound (EUS)-guided Fine-Needle Aspiration (FNA) of the pancreatic mass was performed revealing invasive pancreatic ductal adenocarcinoma incompatible with a prostate cancer metastasis leading to the diagnosis of a PSMA positive pancreatic ductal adenocarcinoma.
\end{abstract}

Keywords: PSMA; pancreatic cancer; PET

An 82-year-old man suffering from prostate cancer was planned for Prostate Specific Membrane Antigen (PMSA) radioligand therapy. The 18F-PSMA PET/CT (Figure 1) scan revealed multilocular PSMA positive tumor lesions (maximum intensity projection; $1 \mathrm{~A}$ ) in the lung (1B), the mediastinal (1B) and the pancreas (1C) as well as in abdominal/iliac lymph nodes (1D). In addition, the patient presented with jaundice and the abdominal ultrasound (US) revealed obstructive extrahepatic cholestasis due to a solid lesion located in the uncinate process of the pancreas. The increased tracer uptake in the uncinate process suggested a prostate cancer metastasis as cause of obstructive jaundice. PSMA uptake in the uncinate process (SUVmax 15; SUVmax: Maximum Standard Uptake Value as a measurement for physiological quantification of radioactivity concentrations) equaled uptake in lymph nodes (SUVmax up to 17) and was even higher than the pulmonary metastasis (SUVmax 7). To exclude secondary malignancy in the pancreatic head, the prostate cancer multidisciplinary team asked for an Endoscopic Ultrasound (EUS) guided biopsy of the uncinate process. The cytoblock preparation showed an invasive pancreatic ductal adenocarcinoma incompatible with a prostate cancer metastasis leading to the diagnosis of a PSMA positive pancreatic ductal adenocarcinoma. PSMA is not only expressed in prostate cancer cells but also in the neovascular endothelium of various solid malignancies possibly due to tumor-associated angiogenic factors and endothelial cell sprouting [1,2].

Numerous solid tumor entities including renal cell carcinoma, thyroid cancer, or Hepatocellular Carcinoma (HCC) showed an increased uptake on 18F-PSMA PET/CT [3-5]. Therefore, it appears that increased PSMA expression is often associated with poorer prognosis [6]. In pancreatic cancer increased PSMA expression is associated with increased tumor aggressiveness in terms of tumor cell proliferation, tumor growth and a reduced overall survival [7]. Some years ago, except for prostate cancer, PSMA avid lesions were described as random findings. The method is now finding its way more and more outside 
prostate cancer imaging [8]. Further studies are required to understand the added value of PSMA PET/CT in non-prostatic pathologies [9].

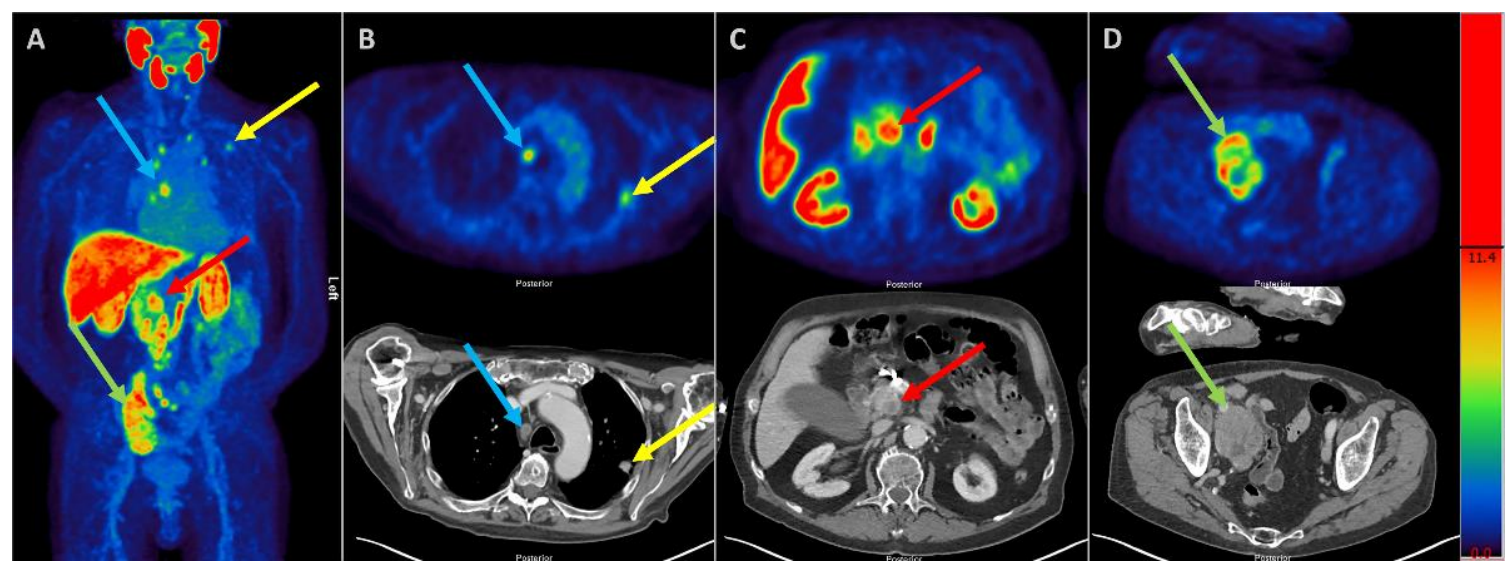

Figure 1. 18F-PSMA PET/CT scan of a patient with known prostate cancer (maximum intensity projection (A)). Multilocular PSMA positive tumor lesions were revealed in the lung (B), the mediastinal (B), the pancreas (C) as well as in abdominal/iliac lymph nodes (D).

Author Contributions: S.S., A.T. acquired data and wrote the paper. J.M., H.I., M.Z. and P.B. critically revised manuscript. All authors have read and agreed to the published version of the manuscript.

Funding: This research received no external funding.

Conflicts of Interest: The authors declare no conflict of interest.

Consent for Publication: The patient provided their written informed consent for the publication of the case.

\section{References}

1. Chang, S.S.; E Reuter, V.; Heston, W.D.; Bander, N.H.; Grauer, L.S.; Gaudin, P.B. Five different anti-prostate-specific membrane antigen (PSMA) antibodies confirm PSMA expression in tumor-associated neovasculature. Cancer Res. 1999, 59, 3192-3198.

2. Chen, W.; Lee, Z.; Awadallah, A.; Zhou, L.; Xin, W. Peritumoral/vascular expression of PSMA as a diagnostic marker in hepatic lesions. Diagn. Pathol. 2020, 15, 1-7. [CrossRef]

3. Mittlmeier, L.M.; Unterrainer, M.; Todica, A.; Cyran, C.C.; Rodler, S.; Bartenstein, P.; Stief, C.G.; Ilhan, H.; Staehler, M. PSMA PET/CT for tyrosine-kinase inhibitor monitoring in metastatic renal cell carcinoma. Eur. J. Nucl. Med. Mol. Imaging 2020, 47, 2216-2217. [CrossRef]

4. Lütje, S.; Gomez, B.; Cohnen, J.; Umutlu, L.; Gotthardt, M.; Poeppel, T.D.; Bockisch, A.; Rosenbaum-Krumme, S. Imaging of Prostate-Specific Membrane Antigen Expression in Metastatic Differentiated Thyroid Cancer Using 68Ga-HBED-CC-PSMA PET/CT. Clin. Nucl. Med. 2017, 42, 20-25. [CrossRef]

5. Kesler, M.; Levine, C.; Hershkovitz, D.; Mishani, E.; Menachem, Y.; Lerman, H.; Zohar, Y.; Shibolet, O.; Even-Sapir, E. 68Ga-PSMA is a novel PET-CT tracer for imaging of hepatocellular carcinoma: A prospective pilot study. J. Nucl. Med. 2019, 60, 185-191. [CrossRef]

6. Jiao, D.; Li, Y.; Yang, F.; Han, D.; Wu, J.; Shi, S.; Tian, F.; Guo, Z.; Xi, W.; Li, G.; et al. Expression of Prostate-Specific Membrane Antigen in Tumor-Associated Vasculature Predicts Poor Prognosis in Hepatocellular Carcinoma. Clin. Transl. Gastroenterol. 2019, 10, e00041-e00047. [CrossRef] [PubMed]

7. Ren, H.; Zhang, H.; Wang, X.; Liu, J.; Yuan, Z.; Hao, J. Prostate-specific membrane antigen as a marker of pancreatic cancer cells. Med Oncol. 2014, 31, 1-6. [CrossRef] [PubMed]

8. Patel, D.; Loh, H.; Le, K.; Stevanovic, A.; Mansberg, R. Incidental Detection of Hepatocellular Carcinoma on 68Ga-Labeled Prostate-Specific Membrane Antigen PET/CT. Clin. Nucl. Med. 2017, 42, 881-884. [CrossRef] [PubMed]

9. Thakar, A.; Sakthivel, P.; Arunraj, S.T.; Bhalla, A.S.; Kakkar, A.; Kumar, R. Comparison of Prostate-Specific Membrane Antigen PET/CT and Contrast-Enhanced Magnetic Resonance Imaging in Follow-up Assessment of Juvenile Nasal AngiofibromaA Novel Pilot Study. Clin. Nucl. Med. 2020, 45, e498-e504. [CrossRef] [PubMed] 